\section{PERITZ: A FORTRAN program for performing multiple comparisons of means using the Peritz $\mathbf{Q}$ method}

\section{SAMUEL A. MARTIN and LARRY E. TOOTHAKER University of Oklahoma, Norman, Oklahoma}

Several methods have been proposed for the comparison of means (see Miller, 1981; or Hochberg \& Tamhane, 1987). Multiple comparison procedures for the completely randomized univariate design are available in commercial statistics packages such as SAS, SPSS ${ }^{\mathbf{x}}$, and BMDP. However, the analysis of mean differences in a design more sophisticated than the completely randomized univariate design requires extra programming in these packages-when it is at all possible. In two-way and higher order ANOVA designs, the most frequent approach to hypothesis testing is to test for overall significance by using the ANOVA followed by a comparison of cell means when there is a significant interaction, or by a comparison of marginal means when a main effect is significant. Cell mean comparisons are not handled in a straightforward manner in these packages. In addition, empirical research into the performance of multiple comparison procedures (Einot \& Gabriel, 1975; Ramsey, 1978, 1981) indicates that most range procedures available in the statistics packages either are too liberal in attempting to control for Type I errors or are lacking in power. (See Jaccard, Becker, \& Wood, 1984, for a review of Monte Carlo analysis of multiple comparison procedures.)

All statistics used in hypothesis testing similar to multiple comparison procedures seek to establish the following properties: First, the Type I error rate (probability of falsely rejecting at least one hypothesis of equality of means in an experiment) should not exceed the nominal alpha rate. Second, the multiple comparison procedure should detect as many true differences as possible (power), given that it meets the first criterion of controlling the Type I error rate. Fisher's $L S D$, Duncan's multiple range test, and the Newman-Keuls procedure violate the first requirement, while Scheffé's $S$ procedure and Tukey's $H S D$ protect against Type I errors at the expense of power. The RMP procedure proposed by Ryan (1960) and modified by Einot and Gabriel (1975) and Welsch (1977) (SAS's REGWQ procedure) changes the alpha rate of the Newman-Keuls procedure to protect against the type of configuration where the nominal alpha rate is exceeded (multiple equality of means in an experiment in which the full null condition is not true). Where Newman-Keuls uses the studentized range value at level $\alpha$ for each stretch in a stepdown fashion, Ryan's modified procedure allocates

Correspondence may be addressed to Samuel A. Martin, Department of Psychology, 455 West Lindsey, Room 705, Norman, Oklahoma 73019. $\alpha$ among $k$ means for any stretch size $p$ at $1-(1-\alpha)^{\mathrm{p} / \mathrm{k}}$ for all stretches of means from size $p=2$ to size $k-2$, and at $\alpha$ for $k-1$ and $k$ stretches. Peritz (Peritz, 1970; see also Begun \& Gabriel, 1981) proposed a method to take advantage of the power of the Newman-Keuls while protecting against the type of mean configuration that provides a problem for this procedure. The Peritz logic involves a stepdown procedure that (1) accepts as nonsignificant any mean difference that Newman-Keuls fails to reject, (2) declares significant any mean difference that is significant at the RMP's alpha level and (3) declares any mean comparison not handled by (1) or (2) as contentious. Contentious sets of means are declared significant if all complementary sets of means are significant using the RMP's alpha level; otherwise, the set is stepdown accepted. Stepdown acceptance involves implicitly declaring as nonsignificant all comparisons of means contained within the stretch of a comparison that has been explicitly declared nonsignificant. Since $\alpha$ rates for stretch sizes $k$ and $k-1$ are equal for both the Newman-Keuls and the RMP procedures, all three procedures are equivalent for a comparison of three means.

The obtaining of critical values at the Ryan's alpha level and the performing of tests on complements of sets of means make the Peritz technique very difficult to compute by hand, and thus it is problematic as a useful method. The program discussed in this paper performs a Peritz pairwise comparison of means. Input to the program (see Appendix A for a sample run) includes the number of groups or cells, the mean square within (error) term, the degrees of freedom within (error), the alpha rate to use for the comparisons, a label for each group to provide group or cell information on the printout, the means to be compared, and the number of subjects per group or cell. By allowing the user to enter the error term and degrees of freedom, this program can be used for repeated measures designs, between-within designs, analysis of covariance designs, and randomized blocks designs. Designs involving unequal numbers of subjects per group or cell use the Tukey-Kramer adjustment procedure (Kramer, 1956; Tukey, 1953), since it has been demonstrated that using harmonic means (as done in SAS, SPSS $^{x}$, and BMDP) can result in a liberal (i.e., excessive Type I error rate) test (Keselman, Toothaker, \& Shooter, 1975).

The program uses a routine published by Dunlap, Powell, and Konnerth (1977) to provide for calculation of exact probabilities for studentized range values. This program supplements the POSTHOC program (Cooksey, 1979) by providing the Peritz procedure as well as removing the requirement that the user enter studentized range values.

The Peritz program was written for a VAX 8600 computer using the VAX/VMS operating system. The program is written in the VAX-FORTRAN implementation 
of the FORTRAN-77 language standard and should be fully compatible with any FORTRAN-77 level compiler. The listing for this program may be found in Appendix B.

\section{REFERENCES}

Begun, J., \& Gabriel, K. R. (1981). Closure of the Newman-Keuls multiple comparisons procedure. Journal of the American Statistical Association, 76, 241-245.

COOKSEY, R. W. (1979). POSTHOC: A FORTRAN program for conducting post hoc multiple comparisons among means. Behavior Research Methods \& Instrumentation, 11, 601.

Dunlap, W. P., Powell, R. S., \& Konnerth, T. K. (1977). A FORTRAN IV function for calculating probabilities associated with the studentized range statistic. Behavior Research Methods \& Instrumentation, 9, 373-375.

Einot, I., \& Gabriel, K. R. (1975). A study of the power of several methods in multiple comparisons. Journal of the American Statistical Association, 70, 574-583.

HoChBERG, Y., \& TAMHANE, A. C. (1987). Multiple comparison procedures. New York: Wiley.

JaCCARD, J., BeCKER, M. A., \& WoOd, G. (1984). Pairwise multiple comparison procedures: A review. Psychological Bulletin, 96, 589-596.

Keselman, H. J., Toothaker, L. E., \& Shooter, M. (1975). An evaluation of two unequal $\mathbf{n}_{\mathbf{k}}$ forms of the Tukey multiple comparison statistic. Journal of the American Statistical Association, 70, 584-587.

KrAMER, C. Y. (1956). Extension of multiple range test to group means with unequal numbers of replications. Biometrics, 13, 307-310.

Miller, R. G. (1981). Simultaneous statistical inference (2nd ed.). New York: McGraw-Hill.

Peritz, E. (1970). A note on multiple comparisons. Unpublished manuscript, Hebrew University, Jerusalem.

Ramsey, P. H. (1978). Power differences between pairwise multiple comparisons. Journal of the American Statistical Association, 73, 479-485.

RamSey, P. H. (1981). Power of univariate pairwise multiple comparison procedures. Psychological Bulletin, 90, 352-366.

RYAN, T. A. (1960). Significance tests for multiple comparisons, variances, and other statistics. Psychological Bulletin, 57, 318-328.

TukEY, J. W. (1953). The problem of multiple comparisons. Unpublished manuscript, Princeton University, Princeton, NJ.

WELSCH, R. E. (1977). Stepwise multiple comparison procedures. Journal of the American Statistical Association, 72, 566-575.

\section{APPENDIX A}

\section{Sample Run of Peritz Program}

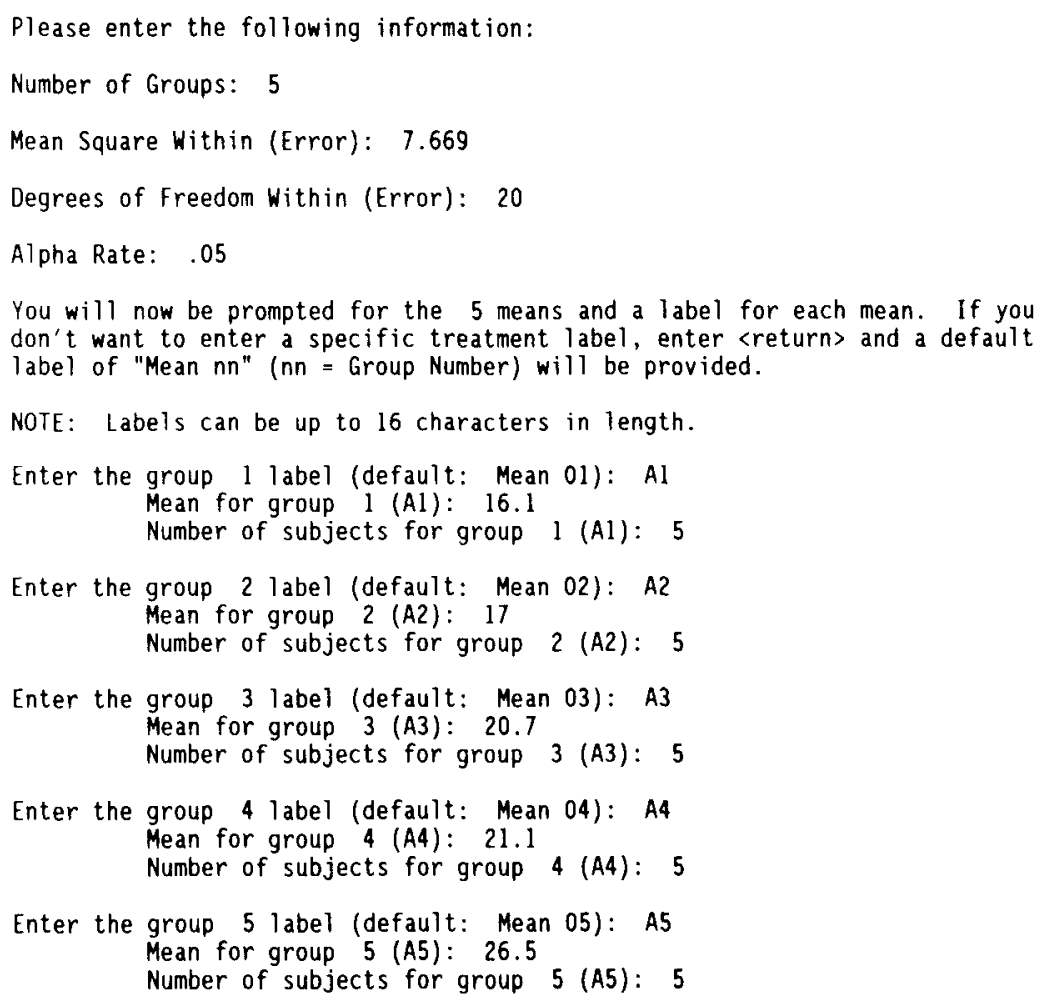

(Program Output)

Number of groups: 5

Mean Square Within:
Degrees of Freedom Within: 20

Set Alpha: 0.050

$\begin{array}{lcccr}\text { Comparison } & \text { Mean } & \text { N } & 0 & \begin{array}{r}\text { Mean } \\ \text { Difference }\end{array} \\ \text { A1 } & 16.10000 & 5 & \text { Non-significant } & 0.90000 \\ \text { A2 } & 17.00000 & 5 & \end{array}$


APPENDIX A (Continued)

\begin{tabular}{|c|c|c|c|c|}
\hline $\begin{array}{l}\text { Al } \\
\text { A3 }\end{array}$ & $\begin{array}{l}16.10000 \\
20.70000\end{array}$ & $\begin{array}{l}5 \\
5\end{array}$ & $\begin{array}{r}3.71427 \\
\text { Significant }\end{array}$ & 4.60000 \\
\hline $\begin{array}{l}\text { A1 } \\
\text { A4 }\end{array}$ & $\begin{array}{l}16.10000 \\
21.10000\end{array}$ & $\begin{array}{l}5 \\
5\end{array}$ & $\begin{array}{r}4.03725 \\
\text { Significant }\end{array}$ & 5.00000 \\
\hline $\begin{array}{l}\mathrm{Al} \\
\mathrm{A5}\end{array}$ & $\begin{array}{l}16.10000 \\
26.50000\end{array}$ & $\begin{array}{l}5 \\
5\end{array}$ & $\begin{array}{r}8.39748 \\
\text { Significant }\end{array}$ & 10.40000 \\
\hline $\begin{array}{l}\text { A2 } \\
\text { A3 }\end{array}$ & $\begin{array}{l}17.00000 \\
20.70000\end{array}$ & $\begin{array}{l}5 \\
5\end{array}$ & $\begin{array}{r}2.98756 \\
\text { Non-significant }\end{array}$ & 3.70000 \\
\hline $\begin{array}{l}\text { A2 } \\
\text { A4 }\end{array}$ & $\begin{array}{l}17.00000 \\
21.10000\end{array}$ & $\begin{array}{l}5 \\
5\end{array}$ & $\begin{array}{r}3.31054 \\
\text { Non-significant }\end{array}$ & 4.10000 \\
\hline $\begin{array}{l}\text { A2 } \\
\text { A5 }\end{array}$ & $\begin{array}{l}17.00000 \\
26.50000\end{array}$ & $\begin{array}{l}5 \\
5\end{array}$ & $\begin{array}{r}7.67077 \\
\text { Significant }\end{array}$ & 9.50000 \\
\hline $\begin{array}{l}\text { A3 } \\
\text { A4 }\end{array}$ & $\begin{array}{l}20.70000 \\
21.10000\end{array}$ & $\begin{array}{l}5 \\
5\end{array}$ & $\begin{array}{r}0.32298 \\
\text { Non-significant }\end{array}$ & 0.40000 \\
\hline $\begin{array}{l}\text { A3 } \\
\text { A5 }\end{array}$ & $\begin{array}{l}20.70000 \\
26.50000\end{array}$ & $\begin{array}{l}5 \\
5\end{array}$ & $\begin{array}{r}4.68321 \\
\text { Significant }\end{array}$ & 5.80000 \\
\hline $\begin{array}{l}\text { A4 } \\
\text { A5 }\end{array}$ & $\begin{array}{l}21.10000 \\
26.50000\end{array}$ & $\begin{array}{l}5 \\
5\end{array}$ & $\begin{array}{r}4.36023 \\
\text { Significant }\end{array}$ & 5.40000 \\
\hline
\end{tabular}

APPENDIX B

Program Peritz

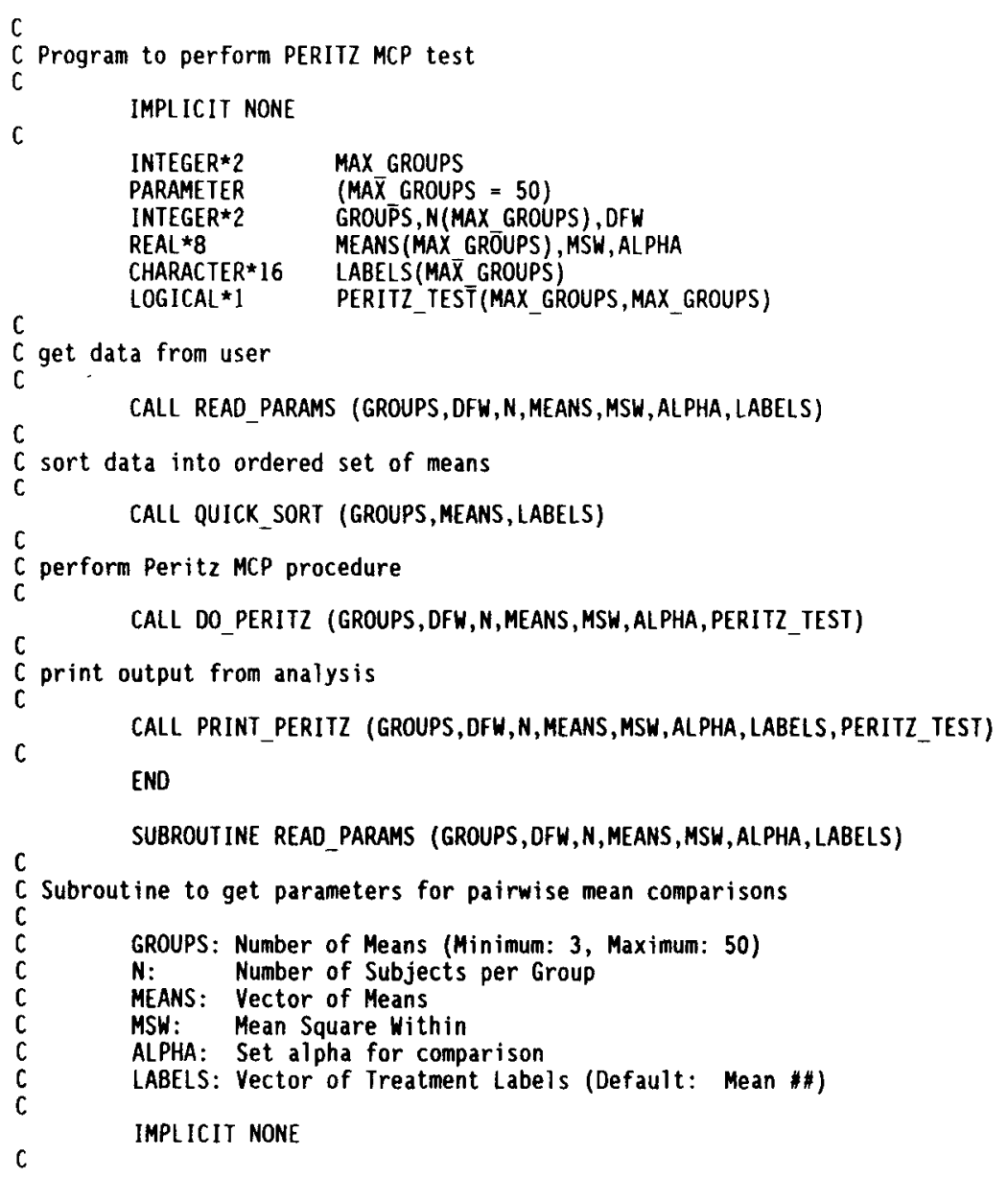


APPENDIX B (Continued)

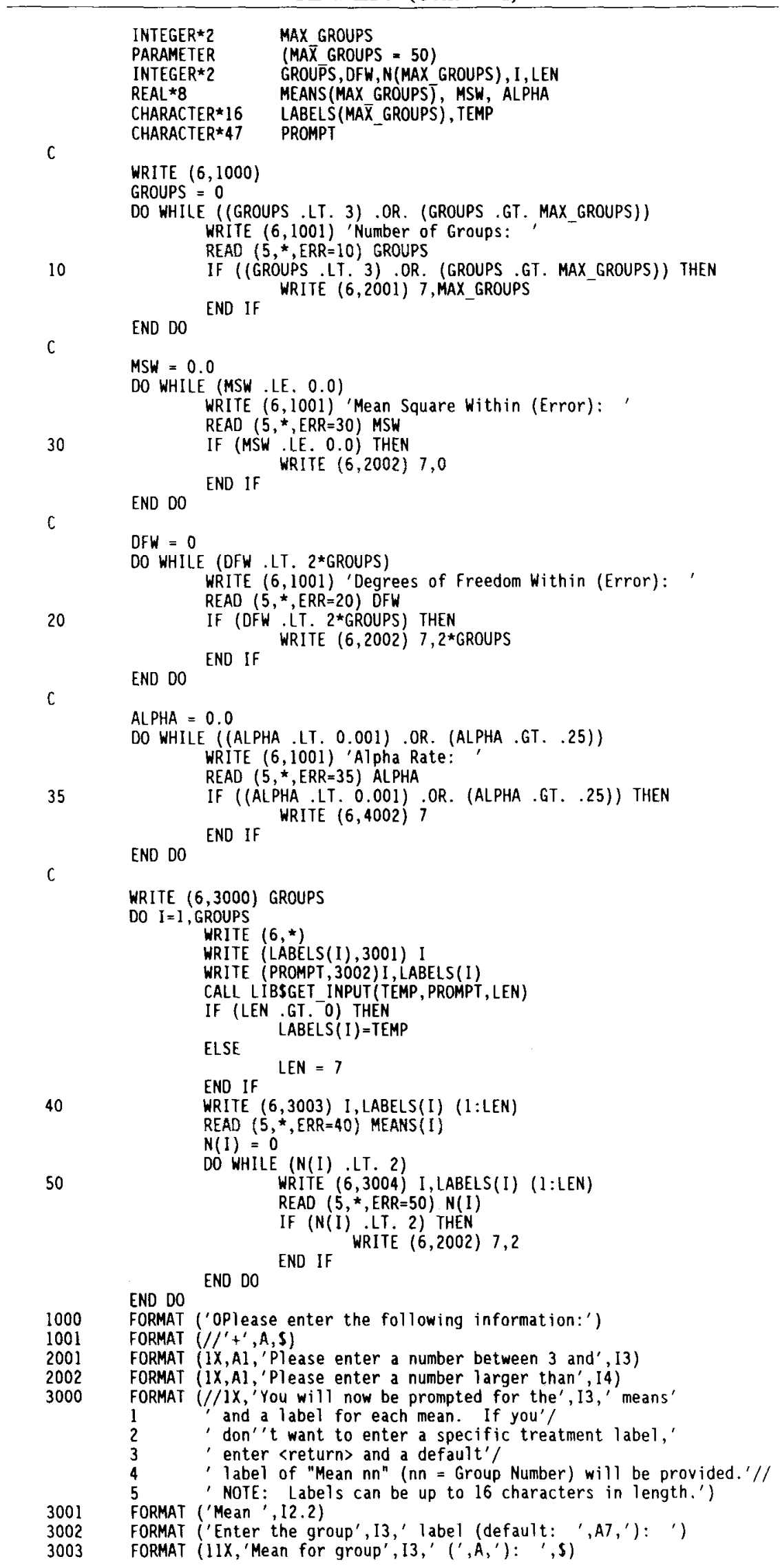




\section{APPENDIX B (Continued)}

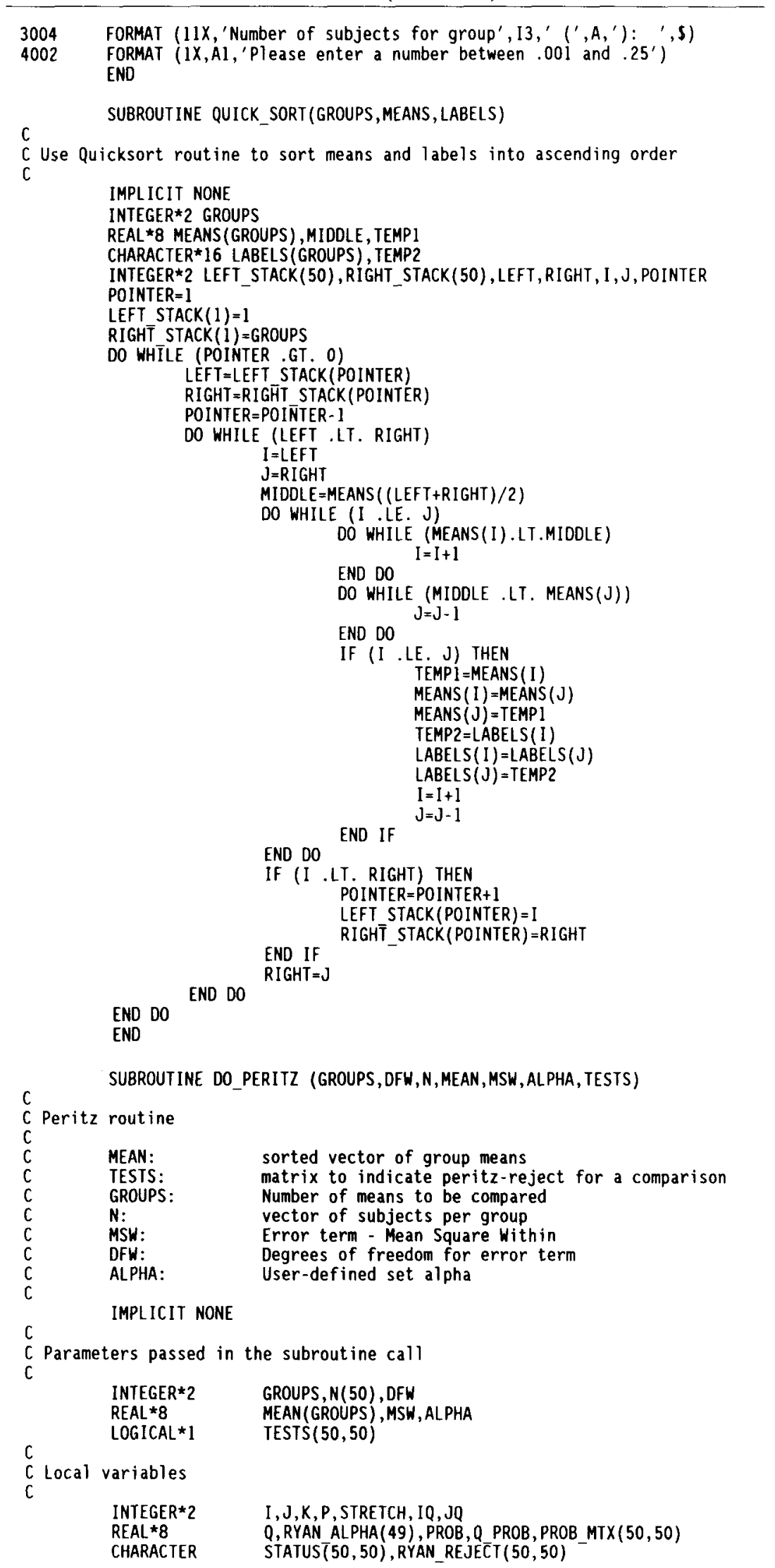


C

C Calculate Ryan's Alpha for each stretch size

$\mathrm{C}$

C

RYAN_ALPHA(GROUPS) = ALPHA

RYAN ${ }^{-}$ALPHA (GROUPS-1) = ALPHA

DO I $=2$, GROUPS -2

END DO

RYAN_ALPHA $(I)=1-(1-\text { ALPHA })^{\star \star}($ FLOAT(I) $/$ FLOAT (GROUPS) $)$

C Set up test matrix to initially show that all comparisons are Peritz reject

C Set up status matrix to indicate that all comparisons are NK-reject

c Set up matrix to indicate if any pw-comparison is Ryan-significant

C

DO I $=1$, GROUPS

DO $\mathrm{J}=1$, GROUPS

$\operatorname{TESTS}(I, J)=. \operatorname{TRUE}$.

$\operatorname{STATUS}(I, J)={ }^{\prime} C^{\prime}$

END DO

RYAN_REJECT $(I, J)={ }^{\prime} R^{\prime}$

END D0

$$
\text { c }
$$

C Do STP comparison of all means to test if Ryan critical

C

DO $I=1$, GROUPS -1

DO $\mathrm{J}=\mathrm{I}+\mathrm{I}$, GROUPS

STRETCH $=\mathrm{J}-\mathrm{I}+1$

$Q=A B S(\operatorname{MEAN}(J)-\operatorname{MEAN}(\mathrm{I})) / \operatorname{SQRT}((\operatorname{MSW} / N(\mathrm{I})+\operatorname{MSW} / N(\mathrm{~J})) / 2$.)

$P R O B=Q$ PROB ( $Q$, STRETCH, DFW)

PROB MT $X(I, J)=P R O B$

END DO

IF (PROB .GT. RYAN_ALPHA(STRETCH)) RYAN_REJECT $(I, J)=$ ' $A$ '

END DO

$$
\text { C }
$$

C Perform the Newman-Keuls stepdown procedure

C Note: An NK accept is an automatic Peritz accept

C

STRETCH = GROUPS

DO WHILE (STRETCH . GE. 2)

DO I $=1$, GROUPS-STRETCH +1

$\mathrm{P}=\mathrm{I}+\mathrm{STRETCH}-1$

IF (TESTS $(I, P)$ ) THEN

C IF not NK-critical, stepdown accept

C

IF (PROB MTX( $I, P)$.GT.ALPHA) THEN

DO $\mathrm{J}=\overline{\mathrm{I}}, \mathrm{P}-\mathrm{I}$

DO $K=J+1, P$

$\operatorname{TESTS}(J, K)=$. FALSE.

END DO

END DO

C

C If Ryan-critical, reject

C

ELSE IF (RYAN REJECT(I,P).EQ. 'R') THEN

$c$ $\operatorname{STATUS}(I, P)={ }^{\prime} R^{\prime}$

$C$ Otherwise, perform the Peritz procedure

C

C

ELSE

C Test the complements to the left

C

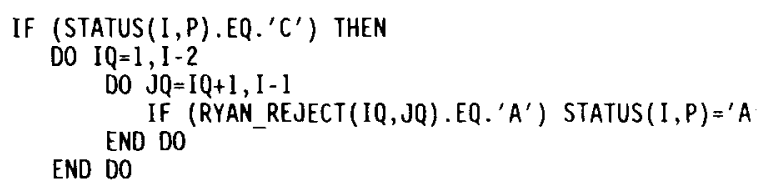

END IF

$\mathrm{C}$

C Test the complements to the right

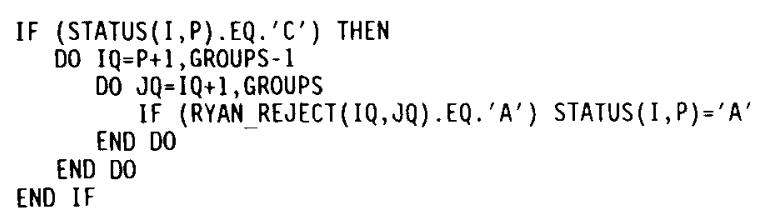


APPENDIX B (Continued)

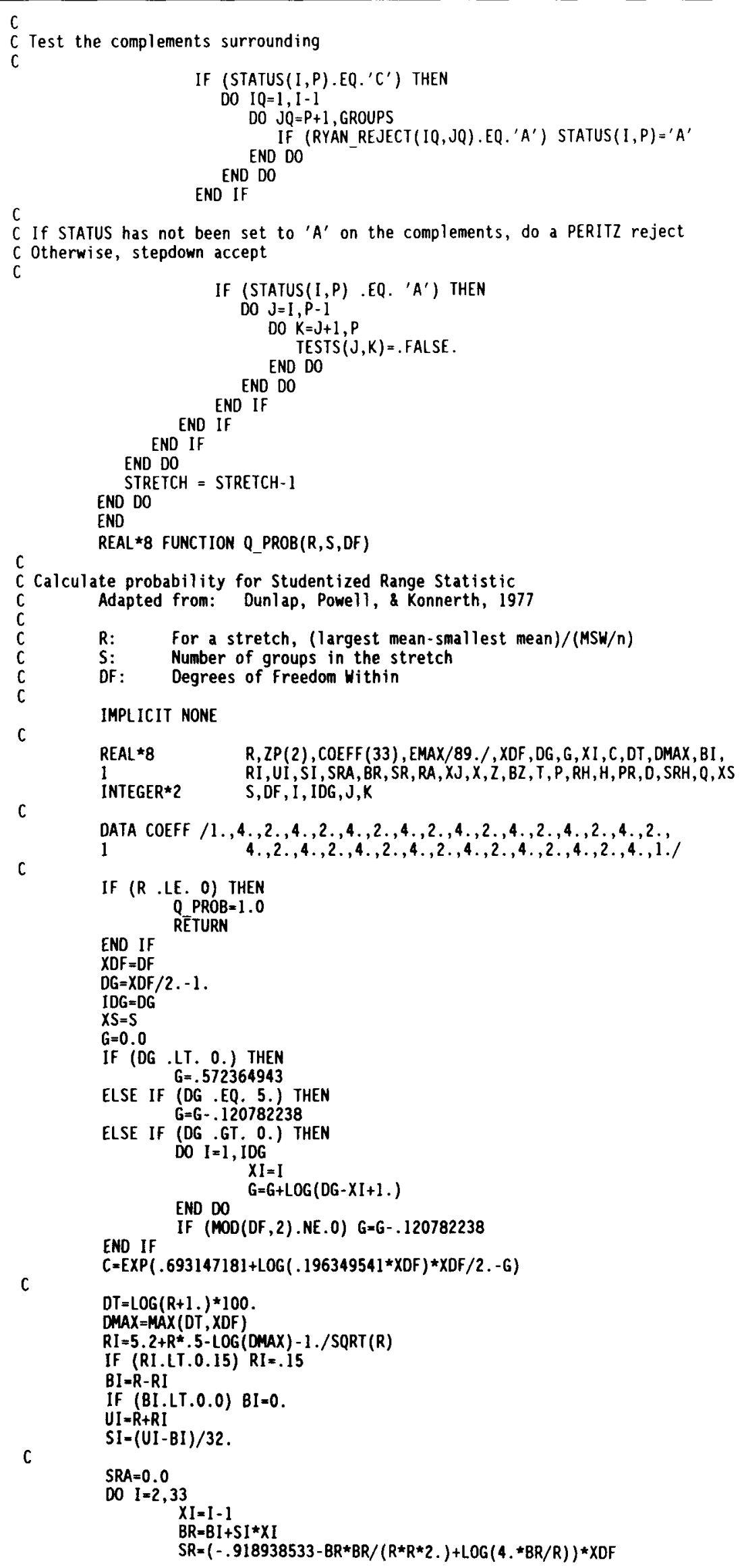


c

$R A=0.0$

$D O \mathrm{~J}=1,33$

$\mathrm{XJ}=\mathrm{J}-1$

$B Z=X J * .34375-7$.

$\mathrm{Z}=\mathrm{BR}+\mathrm{BZ}$

DO $K=1,2$

IF (K.EQ.2) $Z=B Z$

$X=A B S(Z)$

$Q=.39894228 * \operatorname{EXP}(-X * X / 2$.

IF $(X$.GT. 3.7) THEN

ELSE $Z \dot{P}(K)=Q *(\operatorname{SQRT}(4 .+X \star X)-X) / 2$.

$T=1 . /(1 .+.2316419 * \mathrm{x})$

$P=.31938153 * T$

$P=P-.356563782 \star T \star \star 2$

$P=P+1.78147937 * T * \star 3$

$P=P-1.821255978 * T * \star 4$

$P=P+1.330274429 \star T \star \star 5$

END IF

$Z P(K)=Q \star P$

END DO

IF (Z .GT. 0.) $Z P(K)=1 .-Z P(K)$

$\mathrm{RH}=0$.

$D=Z P(1)-Z P(2)$

IF (D .GT. O) THEN

$H=\operatorname{LOG}(D)$

$P R=-.918938533-B Z \star B Z / 2 .+H *(X S-1)+.L O G(X S)$

END IF

IF (PR+EMAX.GT.0) RH=EXP(PR)

END DO

$R A=R A+\operatorname{COEFF}(\mathrm{J}) * R H$

$R A=.34375 \star R A / 3$.

$\mathrm{SRH}=0$.

$D=(1 .-R A) / B R$

IF (D .GT. 0.) THEN

IF $(S R+E M A X . G T .0) \quad S R H=E X P(S R)$

END IF

$S R A=S R A+\operatorname{COEFF}(I) \star S R H$

END DO

$Q \mathrm{PROB}=S I * S R A / 3 .{ }^{*} \mathrm{C}$

END

SUBROUTINE PRINT_PERITZ (GROUPS, DFW, N, MEANS, MSW, ALPHA, LABELS, PERITZ_TEST)

$c$

$C$ Subroutine to print results from PERITZ MCP analysis

c

c

IMPLIC IT NONE

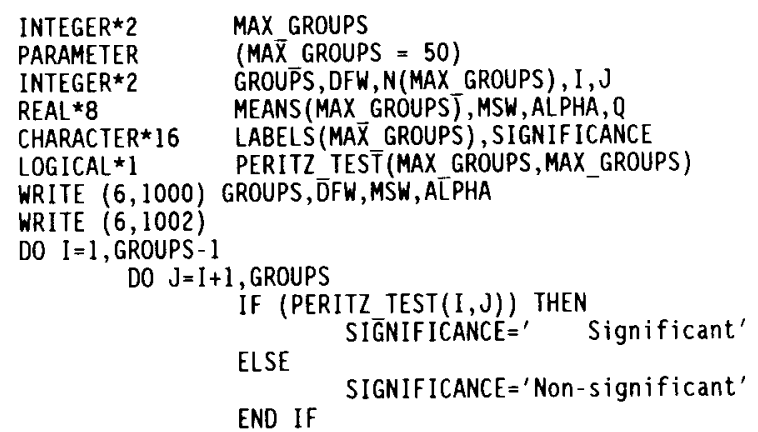

$Q=\operatorname{ABS}(\operatorname{MEanS}(\mathrm{I})-\operatorname{MEANS}(\mathrm{J})) / \operatorname{SQRT}((\operatorname{MSW} / \mathrm{N}(\mathrm{I})+\operatorname{MSW} / \mathrm{N}(\mathrm{J})) / 2$.

$\frac{1}{2}$

WRITE $(6,1001)$

LABELS(I), MEANS(I), N(I), Q, MEANS(J)-MEANS(I), LABELS(J), MeANS(J),

END DO $\mathrm{N}(\mathrm{J})$, SIGNIF ICANCE

END DO

1000

FORMAT

1 (5X, 'Mean Square Within:' 'Fi5, 5 , 21X,'Set Alpha:',F6.3/)

1001

FORM

FORMAT

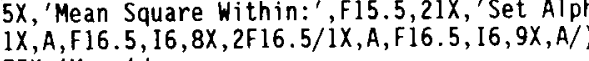

1002

1

75X 'Mean')

END

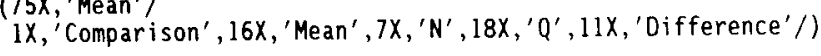

(Manuscript received December 9, 1988;

revision accepted for publication April 21, 1989.) 